\title{
Transfer results for hybrid logic \\ Part I: the case without satisfaction operators
}

\author{
Nick Bezhanishvili and Balder ten Cate
}

February 26, 2004

\begin{abstract}
For every Kripke complete modal logic $L$ we define its hybrid companion $L_{H}$. For a reasonable class of logics, we present a satisfiability-preserving translation from $L_{H}$ to $L$. We prove that for this class of logics, complexity, (uniform) interpolation, finite axiomatization transfer from $L$ to $L_{H}$.
\end{abstract}

\section{Introduction}

Hybrid logic is the result of enriching the modal language with nominals, i.e., symbols that are used to refer to individual points in a Kripke frame. For every Kripke complete modal logic $L$ we define its hybrid companion $L_{H}$ as the hybrid logic of the class of frames defined by $L$. This paper addresses the following question, raised by Gargov and Goranko [7]:

If a modal logic $L$ has a property $\mathfrak{P}$, does its hybrid companion $L_{H}$ have $\mathfrak{P}$ too?

Gargov and Goranko ask this question in the context of a hybrid language with the universal modality. They show for example that canonicity transfers. In this paper, we will focus on a simpler hybrid language, namely the enrichment of the basic modal language with nominals only. In order to obtain transfer results for the language considered by Gargov and Goranko, one would have to combine the results presented in this paper with transfer results for the addition of the universal modality (cf. Goranko and Passy [11] for some partial results).

For every modal logic $L$ which has a master modality we define a translation $(\cdot)^{*}$ from the language of $L_{H}$ to the language of $L$ and prove that if $L$ admits filtration, then $L \vdash \phi^{*}$ iff $L_{H} \vdash \phi$. To use the terminology of Kracht and Wolter [12] and Goguadze, Piazza and Venema [10], we show that $L$ simulates $L_{H}$ under the interpretation $(\cdot)^{*}$. As a corollary, we obtain that complexity and (uniform) interpolation transfer from $L$ to $L_{H}$ and that $L_{H}$ can be axiomatized by adding one simple axiom scheme to the axiomatization of $L$. We prove similar transfer results for a number of logics without master modality, including the basic modal logic $\mathbf{K}$.

Several authors have observed that if a modal logic $L$ admits filtration, then $L_{H}$ also admits filtration and moreover, if $L$ is in addition finitely axiomatizable, then $L_{H}$ is decidable (see e.g. [7] and Mikulas' theorem [2, Theorem 7.8]). While our simulation argument relies to a large extent on the use of filtration, the results it gives rise to are more fine grained: not only decidability transfers from $L$ to $L_{H}$, but also complexity, (uniform) interpolation and finite axiomatizability.

Our results apply to a number of non-elementary hybrid logics. For example, we show that $\mathbf{G L}_{H}$ and $\mathbf{G r z} \mathbf{z}_{H}$ enjoy a form of uniform interpolation. We also derive short proofs for several results that were proved before by hand, e.g., the complexity of $\mathbf{K}_{H}$ [1] and the complexity and finite axiomatizability of $\mathbf{P D L}_{H}$ [13]. Our results confirm the intuition that adding nominals to a modal logic in many cases does not increase the complexity.

In this part of the paper, we only consider the simplest hybrid language, namely the extension of the basic modal language with nominals. As we will show in Part II, many of the transfer results generalize to hybrid languages with satisfaction operators. 


\section{Preliminaries}

\subsection{Hybrid logic in a nutshell}

The minimal hybrid language is an extension of the basic modal language with nominals (cf. [1] and Chapter 7 of [2]). These are special proposition letters, denoted by $i, j, k \ldots$, that designate unique worlds. Formally, given a countable set of proposition letters PROP, and a countable set of nominals NOM, and a set of modalities MOD, the minimal hybrid language is defined as

$$
\phi::=p|i| \neg \phi|\phi \wedge \psi|\langle a\rangle \phi
$$

where $p \in$ PROP, $i \in$ NOM and $a \in$ MOD. While the frames that we work with are the same as in ordinary modal logic, we put one extra condition on the models: each nominal must be true at a unique point in the model. In other words, a model is a pair $(\mathfrak{F}, V)$, where $\mathfrak{F}$ is a frame and $V$ is a valuation for $\mathfrak{F}$ with $|V(i)|=1$ for all $i \in$ NOM. Apart from this extra requirement, no changes are made to the semantics. In particular, the truth definition for nominals is like that of ordinary proposition letters:

$$
(\mathfrak{F}, V), w \Vdash i \text { iff } w \in V(i)
$$

The singleton requirement on the valuation of nominals gives rise to new validities. For instance $(i \wedge \diamond i \wedge \square p) \rightarrow p$ is valid on all frames (if a point is reflexive and all its successors satisfy $p$, then the point itself satisfies $p$ ). Also, using nominals many frame properties are definable that were not definable in the basic modal language. For instance, irreflexivity is defined by $i \rightarrow \neg \diamond i$ (keep in mind that frame validity for hybrid formulas is defined by universal quantification over hybrid valuations, i.e., valuations that assign to each nominal a singleton set).

Given the presence of nominals, it is very natural to further extend the language with satisfaction operators, allowing one to express that a formula holds at a particular point. However, in this part of the paper we will not consider these satisfaction operators.

\subsection{Hybrid companions of modal logics}

Given a frame class $\mathrm{F}$, we will use $L_{H} \mathrm{~F}$ to denote the set of formulas of the minimal hybrid language that are valid on $\mathrm{F}$ (in other words, the hybrid logic of $\mathrm{F}$ ). $L(\mathrm{~F})$ is the set of modal formulas valid on $\mathrm{F}$.

Now, suppose we are given a Kripke complete modal logic $L$, and let $\operatorname{Fr} L$ be the class of frames on which it is valid (note that $L$ is complete for $\operatorname{Fr} L$ ). We will use $L_{H}$ as a shorthand for $L_{H} \operatorname{Fr} L$, i.e., the hybrid logic of the frame class defined by $L$. We call $L_{H}$ the hybrid companion of the modal $\operatorname{logic} L$.

Notice that this is not the only possible way to define hybrid companions for modal logics. In particular, if a modal logic $L$ is complete for several frame classes, the hybrid logics of these classes (as we will see in the next section) need not be the same, and one could consider the hybrid logic of other frame classes than the one defined by $L$. Nevertheless, our choice seems a very natural one.

The main question we address in this paper is the following:

Which properties of logics are preserved under passage from $L$ to $L_{H}$ ?

Here, we provide a result for a class of logics that includes several well-known non-canonical logics, including PDL, GL and Grz. We show that for this class of logics, complexity, finite axiomatizability, interpolation and uniform interpolation transfer.

The following two sections are not essential for the rest of the paper. They merely provide an intuition on the notion of hybrid companion logics. They also show that modal logic and hybrid logic behave rather differently when it comes to lattices of extensions or finite model property. 


\subsection{The lattice of hybrid logics}

The lattice of hybrid logics is quite different from that of modal logics. This can be illustrated very nicely by considering the hybrid logics extending S5. It is well known (see, e.g., [14]) that the lattice of extensions of $\mathbf{S 5}$ is countable, moreover it forms an $\omega+1$-chain and $\mathbf{S 5}$ is the only non-tabular extension of S5. As we will now show, the lattice of extensions of $\mathbf{S} \mathbf{5}_{H}$ looks quite different.

Theorem 2.1 There are continuum many extensions of S5 and continuum many of them are non-tabular.

Proof: Recall from Scroggs [14] that the formula:

$$
\beta(n)=\bigwedge_{k=1}^{n} \diamond p_{k} \rightarrow \bigvee_{1 \leq k<k^{\prime} \leq n} \diamond\left(p_{k} \wedge p_{k^{\prime}}\right)
$$

is valid on an $\mathbf{S 5}$-frame iff the size of each equivalence class is less than $n$. Similarly, for any $n \in \omega$ consider the formula.

$$
\alpha(n)=\diamond \neg\left(i_{1} \vee \cdots \vee i_{n}\right)
$$

The formula $\alpha(n)$ is valid on an S5-frame iff the size of each equivalence class is greater than $n$.

For any $n \in \omega$, let $\mathfrak{F}_{n}$ be the frame $(\{0, \ldots, n-1\},\{0, \ldots, n-1\} \times\{0, \ldots, n-1\})$. Furthermore, for any set of natural numbers $D \subseteq \omega$, let $L^{D}=L\left(\left\{\mathfrak{F}_{n} \mid n \in D\right\}\right)$ and $L_{H}^{D}=L_{H}\left(\left\{\mathfrak{F}_{n} \mid n \in D\right\}\right)$. It is well known that for every infinite $D \subseteq \omega$ we have $L^{D}=\mathbf{S 5}$. Now we show that the same does not hold for hybrid logics. Take any two distinct sets of natural numbers $D_{1}, D_{2} \subseteq \omega$. Without loss of generality, we can assume that there is an $n \in D_{1}$ such that $n \notin D_{2}$. Now consider the formula $\gamma(n)=\alpha(n) \vee \beta(n)$. From the above, it follows that $\gamma(n)$ is valid on a frame iff no equivalence class has exactly $n$ elements. Therefore, $\gamma(n)$ belongs to the hybrid logic $L_{H}^{D_{2}}$ but it does not belong to $L_{H}^{D_{1}}$. Thus, $D_{1}$ and $D_{2}$ characterize different hybrid logics, which implies that there are continuum many complete hybrid logics extending $\mathbf{S} \boldsymbol{5}_{H}$. Moreover, continuum many of them are non-tabular (i.e. are not characterized by a single finite frame).

Incidentally, S5 is about the simplest example of a modal logic with countably many extensions. This raises the question if there exist hybrid logics with countably many extensions (rather than finitely many or continuum many). While this is an interesting question, we will not pursue it here.

\subsection{Finite model property and filtrations}

Recall from Blackburn et al. [2, Definitions 3.23 and 2.27] that a logic $L$ has the finite frame property if for every formula $\phi$ in the language of $L, L \forall \phi$ implies there is a finite $L$-frame $\mathfrak{F}$ (i.e. $\mathfrak{F} \in \operatorname{Fr} L)$ such that $\mathfrak{F} \forall \forall \phi$; and a logic $L$ has the finite model property if $L \forall \phi$ implies that there is a finite model $\mathfrak{M}$ and a world $w$ such that $\mathfrak{M}$ globally satisfies all formulas in $L$ and $\mathfrak{M}, w \not \models \phi$. It is well-known that these two properties coincide for normal modal logics (cf. Blackburn et al. [2, Theorem 3.28] or Chagrov and Zakharyaschev [5, Theorem 8.47]). In this subsection we show that for hybrid logics these two notions do not coincide in general. However, they do coincide for companion logics.

Theorem 2.2 Finite model property and finite frame property do not coincide for hybrid logics.

Proof: Consider the Kripke frame $\mathfrak{F}_{\omega}=(\omega, \omega \times \omega)$. It is well known that $L\left(\mathfrak{F}_{\omega}\right)=\mathbf{S 5}$. Now we show that $L_{H}\left(\mathfrak{F}_{\omega}\right)$ has the finite model property even though it lacks the finite frame property. First we prove that $L_{H}\left(\mathfrak{F}_{\omega}\right)$ does not have any finite frames. This would imply that it lacks the finite frame property. Let $\mathfrak{F}=(W, W \times W)$ be any single-component S5-frame. Then $\mathfrak{F} \models \alpha(n)$ iff $|W|>n$. Since $\mathfrak{F}_{\omega} \models \alpha(n)$ for any $n \in \omega$, it follows that $L_{H}\left(\mathfrak{F}_{\omega}\right)$ lacks the finite frame property. However, we will show that it has the finite model property. 
Suppose $\phi$ is satisfiable on $\mathfrak{F}_{\omega}$. Then there is a valuation $V$ and a point $w_{0} \in \omega$ such that $\left(\mathfrak{F}_{\omega}, V\right), w_{0} \models \phi$. For any subformula $\diamond \psi \in S u b(\phi)$ or a nominal $i \in S u b(\phi)$, choose a representative $w \in \omega$ with $\left(\mathfrak{F}_{\omega}, V\right), w \models \psi$ (if such point exists) or $\left(\mathfrak{F}_{\omega}, V\right), w \models i$ respectively. Let $S$ be the set of all selected points plus $w_{0}$ and $w_{1}$, for some distinct $w_{1} \in \omega$. Let $\mathfrak{F}_{S}=(S, S \times S)$. Now we define the valuation $V^{\prime}$ on $\mathfrak{F}_{S}$ by putting $V^{\prime}(p)=V(p) \cap S$ for any propositional variable $p$. We do the same for the nominals occurring in $\phi$. For any nominal $j$ not occurring in $\phi$, we let $V^{\prime}(j)=\left\{w_{0}\right\}$. By induction, one can show that $\left(\mathfrak{F}_{S}, V^{\prime}\right), w_{0}=\phi$. It is left to be shown that $\left(\mathfrak{F}_{S}, V^{\prime}\right), w_{0}=L_{H}\left(\mathfrak{F}_{\omega}\right)$.

Let $V^{\prime \prime}$ be a valuation on $\mathfrak{F}_{\omega}$ such that for all proposition letters $p$ and worlds $w \in \omega$,

$$
w \in V^{\prime \prime}(p) \text { iff } \begin{cases}w \in V^{\prime}(p) & \text { in case } w \in S \\ w_{1} \in V^{\prime}(p) & \text { otherwise }\end{cases}
$$

and $V^{\prime \prime}(i)=V(i)$ for all nominals $i$. Then it is easy to see that $\left(\mathfrak{F}_{S}, V^{\prime}\right)$ is bisimilar to $\left(\mathfrak{F}_{\omega}, V^{\prime \prime}\right)$ (the bisimulation is the relation $\left.\{(w, w) \mid w \in S\} \cup\left\{\left(w_{1}, w\right) \mid w \in \omega \backslash S\right\}\right)$. Hence $\left(\mathfrak{F}_{S}, V^{\prime}\right)$ satisfies the same formulas as $\left(\mathfrak{F}_{\omega}, V^{\prime \prime}\right)$ and therefore is a model for $L_{H}\left(\mathfrak{F}_{\omega}\right)$. This finishes the proof of the theorem.

Throughout this paper, we will mainly be concerned with logics that admit filtration. Now we will briefly recall the idea of filtration.

Let $\mathfrak{M}$ be a model based on a frame $\mathfrak{F}=(W, R)$ and let $\Sigma$ be a set of formulas closed under subformulas. Define an equivalence relation $\sim_{\Sigma}$ on $W$ by putting for every $w, v \in W$ and $\psi \in \Sigma$ :

$$
w \sim_{\Sigma} v \quad \text { if } \quad w \models \psi \Leftrightarrow v \mid=\psi
$$

Denote by $[w]$ the $\sim_{\Sigma}$-equivalence class containing $w$ and let $W / \sim_{\Sigma}$ be the set of all $\sim_{\Sigma}$-equivalence classes of $W$. Define a valuation $V_{\Sigma}$ on $W / \sim_{\Sigma}$ by putting $V_{\Sigma}(p)=\{[w]: w \models p\}$. The model $\mathfrak{M} / \sim_{\Sigma}=\left(W / \sim_{\Sigma}, R_{\Sigma}, V_{\Sigma}\right)$ is called a filtration of $\mathfrak{M}$ through $\Sigma$ if $R_{\Sigma}$ is such a binary relation on $W / \sim_{\Sigma}$ that for any $\psi \in \Sigma$ and $w \in W$, we have:

$$
\mathfrak{M}, w \models \psi \quad \text { iff } \quad \mathfrak{M} / \sim_{\Sigma},[w] \models \psi
$$

It is easy to see that $\left|W / \sim_{\Sigma}\right| \leq 2^{|\Sigma|}$. Hence $W / \sim_{\Sigma}$ is finite whenever $\Sigma$ is finite. The frame $\mathfrak{F} / \sim_{\Sigma}=\left(W / \sim_{\Sigma}, R_{\Sigma}\right)$ is called a filtration of $\mathfrak{F}$ through $\Sigma$.

Definition 2.3 We say that a modal logic L admits filtration if for every formula $\phi$ there exists a finite set of formulas $\Sigma_{\phi}$ containing all subformulas of $\phi$ such that for every $L$-frame $\mathfrak{F}=(W, R)$ a point $w \in W$ and a model $\mathfrak{M}=(\mathfrak{F}, V)$ with $\mathfrak{M}, w=\phi$, some filtration of $\mathfrak{F}$ over $\Sigma_{\phi}$ is an $L$-frame.

We say that a logic $L$ admits polynomial filtration if it admits filtration and the size of $\Sigma_{\phi}$ is polynomial in the length of $\phi$. We say that a modal logic admits simple filtration if it admits filtration and for every formula $\phi$ we have $\Sigma_{\phi}=S u b(\phi)$.

Note that since the size of $S u b(\phi)$ is linear in the length of $\phi$, every logic that admits simple filtration admits polynomial filtration.

Theorem 2.4 If L admits (polynomial / simple) filtration, then $L_{H}$ admits (polynomial / simple) filtration. ${ }^{1}$

Proof: We simply apply the usual filtration, treating nominals as proposition letters. All that needs to be checked is that the filtrated model is a hybrid model, in other words, that every nominal occurring in a given formula is true at exactly one point. Since each nominal is true at some point in the original model, it must also be true at some point in the filtrated model. Now, suppose that a nominal $i$ is true at two points of the filtrated model, say $[w]$ and $[v]$. Then $w \models i$ and $v \models i$ in the original model, and so $w=v$, which implies that $[w]=[v]$.

\footnotetext{
${ }^{1}$ Gargov and Goranko $[7, \S 6.3]$ prove a similar result for hybrid logics with the universal modality.
} 
Now we are ready to prove that for the companion logics the notions of the finite frame property and the finite model property do coincide.

Theorem 2.5 A hybrid logic $L_{H}$ of a modally definable class of frames (equivalently, a hybrid companion logic), has the finite model property iff it has the finite frame property.

Proof: The proof is similar to the proof of Theorem 3.28 from Blackburn et al. [2]. The right-toleft implication is straightforward. For the converse, suppose a hybrid companion logic $L_{H}$ has a finite model property. Let $L$ be the corresponding modal logic and $\phi$ a hybrid formula which does not belong to $L_{H}$. Then there is a model $\mathfrak{M}$, such that $\mathfrak{M}$ globally satisfies all formulas of $L_{H}$, and such that $\mathfrak{M}, w \not \models \phi$. Let $\Sigma$ be the closure of the set $\{\phi\} \cup L$ under subformulas. We filtrate $\mathfrak{M}$ through $\Sigma$. Let $\mathfrak{M}^{\prime}=\left(\mathfrak{F}^{\prime}, V\right)$ be the filtrated model. The same arguments as in the proof of $[2$, Theorem 3.28] show that $\mathfrak{F}^{\prime}$ is an $L$-frame, hence it is an $L_{H}$-frame. Theorem 2.4 shows that $\mathfrak{M}^{\prime}$ is a hybrid model (i.e. every nominal is true at exactly one point) and $\mathfrak{M}^{\prime},[w] \forall \phi$. Therefore, $L_{H}$ has a finite frame property.

\section{The translation}

In this section, we present our main result. We define two translations from the minimal hybrid language to the basic modal language, and prove that they preserve satisfiability. The first translation applies to logics with a master modality, and the second one applies to a number of logics without a master modality.

\subsection{Logics with a master modality}

Definition 3.1 A modal logic $L$ has a master modality [2, $p$. 371] if there is a formula $\phi(p)$ containing only the proposition letter $p$ such that for all models $\mathfrak{M}$ based on an L-frame and worlds $w, \mathfrak{M}, w \models \phi(p)$ iff $p$ is true somewhere in the submodel of $\mathfrak{M}$ generated by $w$ (equivalently, if $p$ is true in a point reachable from $w$ in a finite number of steps). If a logic has a master modality, we will refer to it by $\diamond^{+}$(more precisely, we will use $\diamond^{+} \psi$ as a shorthand for $\left.\phi(\psi)\right)$.

Fact 3.2 1. Every logic of bounded depth has a master modality.

2. Every extension of $\mathbf{K} \mathbf{4}$ has a master modality.

3. Every extension of $\mathbf{K 5}$ has a master modality.

4. PDL has a master modality. ${ }^{2}$

5. Every extension of $\mathbf{K} \mathbf{4} \times \mathbf{K} \mathbf{4}$ has a master modality.

6. Every logic with universal modality has a master modality.

7. Every extension of the tense logic $\mathbf{K} \mathbf{4}_{t}$ with trichotomy has a master modality (where trichotomy is the axiom $P p \wedge P q \rightarrow P(p \wedge P q) \vee P(q \wedge P p) \vee P(p \wedge q))$.

Proof: 1 . Take $\phi=\bigvee_{0 \leq k \leq n} \diamond^{k} p$, where $n$ is the bound on the depth.

2. Take $\phi=p \vee \diamond p$.

3. Take $\phi=p \vee \diamond p \vee \diamond \diamond p$

4. Take $\phi=\left\langle\left(\cup_{i} a_{i}\right)^{*}\right\rangle p$.

5. Take $\phi=p \vee \diamond_{1} p \vee \diamond_{2} p \vee \diamond_{1} \diamond_{2} p$.

\footnotetext{
${ }^{2}$ For convenience, we assume that the language contains only finitely many atomic programs. The results of this paper can easily be generalized to PDL with infinitely many atomic programs.
} 
6. Take $\phi=E p$.

7. Take $\phi=p \vee P p \vee P F p$

Note that $\mathbf{K}$ does not have a master modality (this can easily be shown by the fact that every modal formula has a finite modal depth, and hence can only talk about a small part of the generated submodel). Similarly, the basic tense logic $\mathbf{K}_{t}$ and the tense logic of transitive frames $\mathbf{K} \mathbf{4}_{t}$ do not have a master modality.

The class of logics we will be working with is the class of logics having the master modality that admit filtration. Let $L$ be a Kripke complete modal logic with master modality that admits filtration. Now we define a translation from the language of $L_{H}$ to the language of $L$. That is we translate every hybrid formula into a modal formula. For a hybrid formula $\phi\left(i_{1}, \ldots, i_{n}\right)$ let $\phi\left[\vec{i} / \overrightarrow{p_{i}}\right]$ denote the formula obtained from $\phi$ by uniformly replacing each nominal $i_{k}$ by distinct new proposition letter $p_{i_{k}}$. For a hybrid formula $\phi\left(i_{1}, \ldots, i_{n}\right)$, let

$$
\phi^{*}=\phi\left[\vec{i} / \overrightarrow{p_{i}}\right] \wedge \bigwedge_{\substack{\left.1 \leq k \leq n \\ \psi \in \Sigma_{\phi[\vec{i} / \vec{i} \vec{i}}\right]}} \diamond^{+}\left(p_{i_{k}} \wedge \psi\right) \rightarrow \square^{+}\left(p_{i_{k}} \rightarrow \psi\right)
$$

where $\Sigma_{\phi\left[\vec{i} / \overrightarrow{p_{i}}\right]}$ is the filtration set of $\phi\left[\vec{i} / \overrightarrow{p_{i}}\right]$.

Theorem 3.3 Let $L$ be a logic with a master modality that admits filtration. Let $\phi$ be any hybrid formula. Then $\phi$ is $L_{H}$-satisfiable iff $\phi^{*}$ is L-satisfiable.

\section{Proof:}

$[\Rightarrow]$ Suppose $(\mathfrak{F}, V), w \models \phi$ with $\mathfrak{F} \in \operatorname{Fr} L$. Let $V^{\prime}$ be any valuation that agrees with $V$ on all proposition letters occurring in $\phi$, and such that $V^{\prime}\left(p_{i_{k}}\right)=V\left(i_{k}\right)$ for each nominal $i_{k}$. Clearly, $\left(\mathfrak{F}, V^{\prime}\right), w \models \phi\left[\vec{i} / \overrightarrow{p_{i}}\right]$. The truth of the second conjunct of $\phi^{*}$ at $w$ under $V^{\prime}$ follows directly from the fact that $V^{\prime}\left(p_{i_{k}}\right)$ is a singleton set for each $k=1, \ldots, n$.

$[\Leftarrow]$ Suppose $(\mathfrak{F}, V), w \models \phi^{*}$ with $\mathfrak{F}=(W, R) \in \operatorname{Fr} L$. Without loss of generality, we can assume that $\mathfrak{F}$ is generated by $w$ (note that $\phi^{*}$ is a purely modal formula). Our task is to construct a hybrid model satisfying $\phi$.

First, we will filtrate $(\mathfrak{F}, V)$. Let $\Sigma=\Sigma_{\phi\left[\vec{i} / \overrightarrow{p_{i}}\right]}$. Since $L$ admits filtration, there exists a model $\mathfrak{M}=\left(W / \sim_{\Sigma}, R_{\Sigma}, V_{\Sigma}\right)$ such that $\left(W / \sim_{\Sigma}, R_{\Sigma}\right) \in \operatorname{Fr} L$ and such that for all $v \in W$ and $\psi \in \Sigma$, $\mathfrak{M},[v] \models \psi$ iff $(\mathfrak{F}, V), v \models \psi$. In particular, $\mathfrak{M},[w] \models \phi\left[\vec{i} / \overrightarrow{p_{i}}\right]$.

Claim $1 V_{\Sigma}\left(p_{i_{k}}\right)$ contains at most one point (for $\left.k=1, \ldots, n\right)$.

Proof of claim: Suppose $[v],\left[v^{\prime}\right] \in V_{\Sigma}\left(p_{i_{k}}\right)$. Then $v, v^{\prime} \in V\left(p_{i_{k}}\right)$, by the definition of $V_{\Sigma}$. Since $(\mathfrak{F}, V), w \models \diamond^{+}\left(p_{i_{k}} \wedge \psi\right) \rightarrow \square^{+}\left(p_{i_{k}} \rightarrow \psi\right)$ for all $\psi \in \Sigma$, it follows that $v, v^{\prime}$ agree on formulas in $\Sigma$. Indeed, if $v \models \psi$ then $w \models \diamond^{+}\left(p_{i_{k}} \wedge \psi\right)$, so $w \models \square^{+}\left(p_{i_{k}} \rightarrow \psi\right)$ and therefore $v^{\prime} \models \psi$. Thus, $v \sim_{\Sigma} v^{\prime}$ and so $[v]=\left[v^{\prime}\right]$.

If every $p_{i_{k}}$ is true at exactly one point, then the proof is finished, since we can consider $\left(W / \sim_{\Sigma}, R_{\Sigma}\right)$ to be a hybrid model for $\phi$. In general, however, this need not be the case: $p_{i_{k}}$ could be true nowhere. So, we need to ensure that for every $p_{i_{k}}$ there is indeed a point where $p_{i_{k}}$ is true. Let $\mathfrak{G}$ be the disjoint union of two isomorphic copies of $\left(W / \sim_{\Sigma}, R_{\Sigma}\right)$. For convenience, we will use $[v]_{1}$ and $[v]_{2}$ to refer the two distinct copies of a world $[v] \in W / \sim_{\Sigma}$. Since $\operatorname{Fr} L$ is modally definable, it is closed under disjoint unions and hence, $\mathfrak{G} \in \operatorname{Fr} L$. Define the valuation $V^{\prime}$ for $\left(W / \sim_{\Sigma}, R_{\Sigma}\right)$ by putting $V^{\prime}(p)=\left\{v_{1} \mid v \in V_{\Sigma}(p)\right\}$ for each proposition letter $p$ occurring in $\phi$, and for each nominal $k=1, \ldots, n$,

$$
V^{\prime}\left(p_{i_{k}}\right)= \begin{cases}\left\{[v]_{1}\right\} & \text { if } V_{\Sigma}\left(p_{i_{k}}\right)=\{[v]\} \\ \left\{[w]_{2}\right\} & \text { if } V_{\Sigma}\left(p_{i_{k}}\right)=\emptyset\end{cases}
$$


Intuitively speaking, the only role of the second disjoint copy of $\left(W / \sim_{\Sigma}, R_{\Sigma}\right)$ is to provide enough points so that we can make each $p_{i_{k}}$ true somewhere, without affecting the truth of $\phi$ at $[w]$. Indeed, a simple bisimulation argument shows that $\left(\mathfrak{G}, V^{\prime}\right),[w] \models \phi\left[\vec{i} / \vec{p}_{i}\right]$.

By construction, $V^{\prime}$ assigns to each $p_{i_{k}}$ a singleton set. Replacing each $p_{i_{k}}$ by the corresponding $i_{k}$, we therefore obtain a hybrid model again, which furthermore satisfies $\phi$ at $[w]_{1}$. We conclude that $\phi$ is satisfiable on $\operatorname{Fr} L$.

Corollary 3.4 Let L be a complete modal logic with a master modality that admits filtration. Let $\phi$ be any hybrid formula with nominals $i_{1}, \ldots i_{n}$. Then $\phi$ is $L_{H}$-valid iff the formula

$$
\left(\bigwedge_{\substack{1 \leq k \leq n \\ \psi \in \Sigma_{\neg \phi}}} \diamond^{+}\left(p_{i_{k}} \wedge \psi\right) \rightarrow \square^{+}\left(p_{i_{k}} \rightarrow \psi\right)\right) \rightarrow \phi\left[\vec{i} / \overrightarrow{p_{i}}\right]
$$

is L-valid.

Proof: Suppose $L_{H} \vdash \phi$, for some formula $\phi$ with nominals $i_{1}, \ldots, i_{n}$. Then $\neg \phi$ is not $L_{H^{-}}$ satisfiable. Hence, by Theorem 3.3,

$$
\left(\bigwedge_{\substack{1 \leq k \leq n \\ \psi \in \Sigma_{\neg \phi}}} \diamond^{+}\left(p_{i_{k}} \wedge \psi\right) \rightarrow \square^{+}\left(p_{i_{k}} \rightarrow \psi\right)\right) \wedge \neg \phi\left[\vec{i} / \overrightarrow{p_{i}}\right]
$$

is not $L$-satisfiable, which means that

$$
L \vdash\left(\bigwedge_{\substack{1 \leq k \leq n \\ \psi \in \Sigma}} \diamond^{+}\left(p_{i_{k}} \wedge \psi\right) \rightarrow \square^{+}\left(p_{i_{k}} \rightarrow \psi\right)\right) \rightarrow \phi\left[\vec{i} / \overrightarrow{p_{i}}\right]
$$

Remark 3.5 We remark here that many well-known logics are known to have a master modality and admit polynomial filtration. We list some of them with references for the proofs: K4, K45, KD45, S4, S5, K4.2, K4.3, S4.2, S4.3, K5, K4.1, S4.1 [5, §5.3]; GL and PDL [2, §4.8]; S5 $\times$ S5 [6]; Grz [3]. Moreover, all of these logics except K5, K4.1, S4.1, PDL and Grz admit simple filtration.

\subsection{Logics without a master modality}

Now we show that even though the basic modal logic $\mathbf{K}$ does not have a master modality, our transfer results still can be proved for this logic, as well as some slight extensions of it such as $\mathbf{T}$ and $\mathbf{D}$.

For a hybrid formula $\phi\left(i_{1}, \ldots, i_{k}\right)$ let

$$
\begin{aligned}
& \phi^{*} \quad=\quad \phi\left[\vec{i} / \overrightarrow{p_{i}}\right] \wedge \bigwedge_{1 \leq k \leq n} \diamond^{l}\left(p_{i_{k}} \wedge \psi\right) \rightarrow \square^{m}\left(p_{i_{k}} \rightarrow \psi\right) \\
& \psi \in \operatorname{Sub}\left(\phi\left[\vec{i} / \overrightarrow{p_{i}}\right]\right) \\
& 0 \leq l, m \leq m d(\phi)
\end{aligned}
$$

where $m d(\phi)$ is the modal depth of $\phi[2$, Definition 2.28].

Theorem 3.6 A hybrid formula $\phi$ is $\mathbf{K}_{H}$-satisfiable iff its modal translation $\phi^{*}$ is $\mathbf{K}$-satisfiable.

Proof: The left to right implication is easy to prove. Now suppose that $\phi^{*}$ is satisfiable. Let $\mathfrak{M}, w \models \phi^{*}$, with $\mathfrak{M}=(\mathfrak{F}, V)$ and $\mathfrak{F}=(W, R)$. Without loss of generality, we can assume that $\mathfrak{F}$ is generated by $w$. For every point $v \in W$, let $d_{\mathfrak{F}}(v)$ be the minimal number of $R$-steps 
in which $v$ is reachable from the root $w$. Consider the equivalence relation $\sim_{S u b\left(\phi\left[\vec{i} / \overrightarrow{p_{i}}\right]\right)}$. Two worlds stand in this equivalence relation if they satisfy the same subformulas of $\phi\left[\vec{i} / \overrightarrow{p_{i}}\right]$. For any $\sim_{S u b\left(\phi\left[\vec{i} / \overrightarrow{p_{i}}\right]\right)}$-equivalence class $[v]$, choose a representative $f[v] \in[v]$ such that for any $v^{\prime} \in[v]$ we have $d_{\mathfrak{F}}(f[v]) \leq d_{\mathfrak{F}}\left(v^{\prime}\right)$. Note that while $f[w]=w$, these representatives are in general not unique. Also note that for every $v \in W$ and $\psi \in S u b\left(\phi\left[\vec{i} / \overrightarrow{p_{i}}\right]\right), \mathfrak{M}, v \models \psi$ iff $\mathfrak{M}, f[v] \models \psi$.

Let $W^{\prime}=\{f[v] \mid v \in W\}$. Define the relation $R^{\prime}$ on $W^{\prime}$ by putting $f[u] R^{\prime} f[v]$ iff there is a $v^{\prime} \in[v]$ with $f[u] R v^{\prime}$. Define a valuation $V^{\prime}$ on $W^{\prime}$ by letting $f[w] \in V^{\prime}(p)$ iff $w \in V(p)$ for all $p \in \operatorname{Sub}\left(\phi\left[\vec{i} / \overrightarrow{p_{i}}\right]\right)$. Let $\mathfrak{F}^{\prime}=\left(W^{\prime}, R^{\prime}\right)$ and $\mathfrak{M}^{\prime}=\left(\mathfrak{F}^{\prime}, V^{\prime}\right)$.

Claim 1 For any $\psi \in \operatorname{Sub}\left(\phi\left[\vec{i} / \overrightarrow{p_{i}}\right]\right)$ and a point $v \in W, \mathfrak{M}, f[v]=\psi \quad$ iff $\mathfrak{M}^{\prime}, f[v] \models \psi$

Proof of claim: By the induction on the complexity of $\psi$. If $\psi$ is a propositional letter, then the claim holds by the definition of $V^{\prime}$. The Boolean cases are obvious. Finally, let $\psi=\diamond \chi$.

$[\Rightarrow]$ Suppose that $\mathfrak{M}, f[v] \models \diamond \chi$. Then there is a point $u \in W$ such that $f[v] R u$ and $\mathfrak{M}, u \models \chi$. Since $\chi \in S u b\left(\phi\left[\vec{i} / \overrightarrow{p_{i}}\right]\right)$ and $u \sim_{S u b\left(\phi\left[\vec{i} / \overrightarrow{p_{i}}\right]\right)} f[u]$, we have that $\mathfrak{M}, f[u] \models \chi$. By the induction hypothesis, it follows that $\mathfrak{M}^{\prime}, f[u] \models \chi$. Finally, we have that $f[v] R^{\prime} f[u]$, by the definition of $R^{\prime}$. Hence, $\mathfrak{M}^{\prime}, f[v]={ }^{\prime} \diamond \chi$.

$[\Leftarrow]$ Suppose that $\mathfrak{M}^{\prime}, f[v] \models \diamond \chi$. Then there is an $f[u] \in W^{\prime}$ such that $f[v] R^{\prime} f[u]$ and $\mathfrak{M}^{\prime}, f[u] \models$ $\chi$. By the induction hypothesis, $\mathfrak{M}, f[u] \models \chi$. Also, by the definition of $R^{\prime}$, there must be a $u^{\prime} \in[u]$ such that $f[v] R u^{\prime}$. Since $\chi \in S u b\left(\phi\left[\vec{i} / \overrightarrow{p_{i}}\right]\right)$ and $u^{\prime} \sim_{S u b\left(\phi\left[\vec{i} / \overrightarrow{p_{i}}\right]\right)} f[u]$, it follows that $\mathfrak{M}, u^{\prime} \models \chi$. We conclude that $\mathfrak{M}, f[v] \models \diamond \chi$.

Let us define $d_{\mathfrak{F}^{\prime}}$ similar to $d_{\mathfrak{F}}$. Note that $\mathfrak{F}^{\prime}$ need not be point-generated anymore. For worlds $f[v] \in W^{\prime}$ that are not reachable from the root $f[w]=w$, let $d_{\mathfrak{F}^{\prime}}(f[v])=\infty$.

Claim $2 d_{\mathfrak{F}}(f[v]) \leq d_{\mathfrak{F}^{\prime}}(f[v])$, for all $v \in W$

Proof of claim: If $d_{\mathfrak{F}^{\prime}}(f[v])=\infty$, the claim obviously holds. Otherwise, the proof proceeds by induction on $d_{\mathfrak{F}^{\prime}}(f[v])$. The base case, with $d_{\mathfrak{F}^{\prime}}(f[v])=0$, only applies if $f[v]=w$, in which case the claim clearly holds. Next, suppose $d_{\mathfrak{F}^{\prime}}(f[v])=n+1$. By definition, there must be a path of the form

$$
f[w]=w \underbrace{\stackrel{R^{\prime}}{\longrightarrow} \cdots \stackrel{R^{\prime}}{\longrightarrow}}_{n \text { steps }} f[u] \stackrel{R^{\prime}}{\longrightarrow} f[v]
$$

It follows that $d_{\mathfrak{F}^{\prime}}(f[u]) \leq n$, and hence by the induction hypothesis, $d_{\mathfrak{F}}(f[u]) \leq d_{\mathfrak{F}^{\prime}}(f[u]) \leq n$. Since $f[u] R^{\prime} f[v]$, by the definition of $R^{\prime}$ we have that there is a $v^{\prime} \in[v]$ such that $f[u] R v^{\prime}$. This implies that $d_{\mathfrak{F}}\left(v^{\prime}\right) \leq n+1$. By the definition of $f$, we know that $d_{\mathfrak{F}}(f[v]) \leq d_{\mathfrak{F}}\left(v^{\prime}\right)$, because $v^{\prime} \in[v]$. Therefore, $\bar{d}_{\mathfrak{F}}(f[v]) \leq n+1$.

Claim 3 For all $k=1 \ldots n$, there is at most one world $f[v] \in W^{\prime}$ such that $d_{\mathfrak{F}^{\prime}}(f[v]) \leq \operatorname{md}(\phi)$ and $\mathfrak{M}^{\prime}, f[v] \models p_{i_{k}}$.

Proof of claim: Suppose $\mathfrak{M}^{\prime}, f[v] \models p_{i_{k}}$ and $\mathfrak{M}^{\prime}, f[u] \models p_{i_{k}}$, with $d_{\mathfrak{F}^{\prime}}(f[v]), d_{\mathfrak{F}^{\prime}}(f[u]) \leq \operatorname{md}(\phi)$. By Claim $2, d_{\mathfrak{F}}(f[v]), d_{\mathfrak{F}}(f[u]) \leq m d(\phi)$. Furthermore, $\mathfrak{M}, f[v] \models p_{i_{k}}$ and $\mathfrak{M}, f[u] \models p_{i_{k}}$. By our initial assumption, $\mathfrak{M}, w \models \phi^{*}$, hence $f[v] \sim_{S u b(\phi)} f[u]$, which implies that $f[v]=f[u]$.

From Claim 1, we immediately deduce that $\mathfrak{M}^{\prime}, w \models \phi\left[\vec{i} / \overrightarrow{p_{i}}\right]$. The valuation of $p_{i_{1}} \ldots p_{i_{n}}$ can be restricted to the worlds with depth $\leq m d(\phi)$ without affecting the truth of $\phi\left[\vec{i} / \overrightarrow{p_{i}}\right]$ at $w$. In this way, we make sure that every $p_{i_{k}}$ is true at at most one world. Finally, applying the same argument as in the proof of Theorem 3.3, we conclude that the original hybrid formula $\phi$ is satisfiable.

Theorem 3.7 Let $L$ be any complete uni-modal logic axiomatizable using only formulas of modal depth $\leq 1$ and/or closed formulas. A hybrid formula $\phi$ is $L_{H}$-satisfiable iff $\phi^{*}$ is L-satisfiable. 
Proof: Let us first consider only logics that can be axiomatized using formulas of modal depth $\leq 1$. We use the same construction as in the proof of Theorem 3.6. It suffices to show that the constructed frame $\mathfrak{F}^{\prime}$ is an $L$-frame. We claim that every formula $\phi$ of modal depth 1 that is satisfiable on $\mathfrak{F}^{\prime}$ is also satisfiable on $\mathfrak{F}$. Let $V$ be a valuation for $\mathfrak{F}^{\prime}$, and let $x \in W^{\prime}$ such that $\left(\mathfrak{F}^{\prime}, V\right), x \models \phi$. Let $V^{\prime}$ be defined such that $v \in V^{\prime}(p)$ iff $f[v] \in V(p)$. We claim that for all formulas $\chi$ with $m d(\chi) \leq 1$ and for all $v \in W,\left(\mathfrak{F}, V^{\prime}\right), f[v] \models \chi$ iff $\left(\mathfrak{F}^{\prime}, V\right), f[v] \models \chi$. This, we prove by induction on $\chi$. The only interesting case is for formulas of the form $\diamond \chi$ with $m d(\chi)=0$.

$[\Rightarrow]$ Suppose $\left(\mathfrak{F}, V^{\prime}\right), f[v] \models \diamond \chi$. Then there is a $u \in W$ such that $f[v] R u$ and $\left(\mathfrak{F}, V^{\prime}\right), u \models$ $\chi$. By induction hypothesis, $\left(\mathfrak{F}^{\prime}, V\right), f[u] \models \chi$. By definition of $R^{\prime}, f[v] R^{\prime} f[u]$. Hence, $\left(\mathfrak{F}^{\prime}, V\right), f[v] \mid=\diamond \chi$.

$[\Leftarrow]$ Suppose $\left(\mathfrak{F}^{\prime}, V\right), f[v] \models \diamond \chi$. Then there is an $f[u] \in W^{\prime}$ such that $\left(\mathfrak{F}^{\prime}, V\right), f[u] \models \chi$ and $f[v] R^{\prime} f[u]$. By definition of $R^{\prime}$, there is a $u^{\prime} \in[u]$ such that $f[v] R u^{\prime}$. By induction hypothesis, $\left(\mathfrak{F}, V^{\prime}\right), u^{\prime} \models \chi$. Hence, $\left(\mathfrak{F}, V^{\prime}\right), f[v] \models \diamond \chi$.

The same construction goes through if, in addition, closed formulas are added as axioms to the logic. In that case, we simply extend the filtration set with these closed formulas and their subformulas. As a result, the filtrated model globally satisfies the closed formulas, which implies that they are valid on the underlying frame.

This covers logics axiomatized using reflexivity $(\square p \rightarrow p)$, totality $(\diamond \top)$ and bounded width $\left(\mathrm{bw}_{n} \equiv \bigwedge_{1 \leq k \leq n} \diamond p_{k} \rightarrow \bigvee_{1 \leq k<l \leq n} \diamond\left(p_{k} \wedge p_{l}\right)\right)$.

Corollary 3.8 Let $L$ be a complete uni-modal logic axiomatizable using formulas with modal depth $\leq 1$ and/or closed formulas. Let $\phi$ be any hybrid formula with nominals $i_{1}, \ldots i_{n}$. Then $\phi$ is $L_{H^{-}}$ valid iff the formula

$$
\left(\bigwedge_{\substack{1 \leq k \leq n \\ \psi \in \Sigma_{\neg \phi} \\ 0 \leq n, m \leq m d(\phi)}} \diamond^{n}\left(p_{i_{k}} \wedge \psi\right) \rightarrow \square^{m}\left(p_{i_{k}} \rightarrow \psi\right)\right) \rightarrow \phi\left[\vec{i} / \overrightarrow{p_{i}}\right]
$$

is L-valid.

Proof: As for Corollary 3.4 .

\section{Applications of the translation}

From Theorem 3.3 and 3.6, together with the observation that for logics admitting polynomial filtration, the length of $\phi^{*}$ is polynomial in the length of $\phi$, we obtain the following transfer result for complexity.

Corollary 4.1 Let $L$ be a complete modal logic satisfying one of the following conditions:

(a) L has a master modality and admits polynomial filtration.

(b) $L$ is axiomatizable using only formulas of modal depth $\leq 1$ and/or closed formulas.

Then $L_{H}$-satisfiability is polynomially reducible to L-satisfiability.

Hence, if a modal logic $L$ satisfies the condition (a) or (b), and if C is any complexity class closed under polynomial reductions, such as NP, PSpAcE, ExpTime, NExpTime, 2ExpTime, etc. , then $L$-satisfiability is in (complete for) $\mathrm{C}$ iff $L_{H}$ is in (complete for) C. Note that complexity does not transfer in general. Areces et al. [1] show that adding nominals to the basic tense logic $\mathbf{K}_{t}$ increases the complexity from PSPACE to ExPTIME.

Next, we will discuss the issue of transfer of interpolation and uniform interpolation. 
Definition 4.2 (Interpolation for hybrid logics) A hybrid logic $L$ has interpolation over proposition letters, if for all formulas $\phi$ and $\psi$ with $L \vdash \phi \rightarrow \psi$, there is a formula $\theta$ such that $L \vdash \phi \rightarrow \theta, L \vdash \theta \rightarrow \psi$ and all proposition letters occurring in $\theta$ occur both in $\phi$ and in $\psi$.

Note that according to this definition, $\theta$ might contain nominals occurring in $\phi$ but not in $\psi$ or vice versa. It seems more natural to require that also the nominals occurring in the interpolant $\theta$ should occur both in $\phi$ and in $\psi$. However, it turns out that almost all hybrid logics lack this strong form of interpolation [4].

Recall that a modal logic admits simple filtration if it admits filtration and for every formula $\phi$ we have $\Sigma_{\phi}=S u b(\phi)$. For logics admitting simple filtration, interpolation transfers.

Theorem 4.3 Let $L$ be a complete modal logic satisfying one of the following conditions:

(a) L has a master modality and admits simple filtration.

(b) $L$ is axiomatizable using only formulas of modal depth $\leq 1$ and/or closed formulas.

If $L$ has interpolation, then $L_{H}$ has interpolation over proposition letters.

Proof: We only prove (a), since (b) is similar.

Suppose $L_{H} \vdash \phi \rightarrow \psi$. Let $i_{1}, \ldots, i_{n}$ be the nominals occurring in the formula $\phi \rightarrow \psi$, and let $\Sigma=\operatorname{Sub}\left(\neg(\phi \rightarrow \psi)\left[\vec{i} / \overrightarrow{p_{i}}\right]\right)$ By Corollary 3.4 , the following formula is $L$-valid.

$$
\left(\bigwedge_{\substack{1 \leq k \leq n \\ \chi \in \Sigma}} \diamond^{+}\left(p_{i_{k}} \wedge \chi\right) \rightarrow \square^{+}\left(p_{i_{k}} \rightarrow \chi\right)\right) \rightarrow\left(\phi\left[\vec{i} / \overrightarrow{p_{i}}\right] \rightarrow \psi\left[\vec{i} / \overrightarrow{p_{i}}\right]\right)
$$

The antecedent of this formula says that for all $1 \leq k \leq n$, if two worlds $w$ and $w^{\prime}$ in the model both satisfy $p_{i_{k}}$, then $w$ and $w^{\prime}$ satisfy exactly the same formulas in $\Sigma$. Note that every formula in $\Sigma$ is a Boolean combination of subformulas of $\phi\left[\vec{i} / \overrightarrow{p_{i}}\right]$ and $\psi\left[\vec{i} / \overrightarrow{p_{i}}\right]$. Hence, to say that $w$ and $w^{\prime}$ satisfy the same formulas in $\Sigma$ is equivalent to saying that they satisfy the same subformulas of $\phi\left[\vec{i} / \overrightarrow{p_{i}}\right]$ and $\psi\left[\vec{i} / \overrightarrow{p_{i}}\right]$. Therefore, the formula

$$
\left(\bigwedge_{\substack{1 \leq k \leq n \\ \chi \in S u b\left(\phi\left[\vec{i} / \overrightarrow{p_{i}}\right]\right) \cup \operatorname{Sub}\left(\psi\left[\vec{i} / \vec{p}_{i}\right]\right)}} \diamond^{+}\left(p_{i_{k}} \wedge \chi\right) \rightarrow \square^{+}\left(p_{i_{k}} \rightarrow \chi\right)\right) \rightarrow\left(\phi\left[\vec{i} / \overrightarrow{p_{i}}\right] \rightarrow \psi\left[\vec{i} / \overrightarrow{p_{i}}\right]\right)
$$

is semantically equivalent to $(1)$, and hence $L$-valid. It is easy to see that $(2)$ is equivalent to

$$
\begin{aligned}
& \left(\bigwedge_{\substack{1 \leq k \leq n \\
\chi \in S u b\left(\phi\left[\vec{i} / \overrightarrow{p_{i}}\right]\right)}} \diamond^{+}\left(p_{i_{k}} \wedge \chi\right) \rightarrow \square^{+}\left(p_{i_{k}} \rightarrow \chi\right)\right) \wedge \phi\left[\vec{i} / \overrightarrow{p_{i}}\right] \rightarrow \\
& \left(\bigwedge_{\substack{\left.1 \leq k \leq n \\
\chi \in \operatorname{Sub}\left(\psi \vec{i} / \overrightarrow{p_{i}}\right]\right)}} \diamond^{+}\left(p_{i_{k}} \wedge \chi\right) \rightarrow \square^{+}\left(p_{i_{k}} \rightarrow \chi\right)\right) \rightarrow \psi\left[\vec{i} / \overrightarrow{p_{i}}\right] \\
& \qquad
\end{aligned}
$$

Let $\theta$ be the modal interpolant for $(3)$ in $L$. Note that apart from the proposition letters $p_{i_{1}}, \ldots p_{i_{n}}$, $\theta$ only contains proposition letters that occur both in $\phi$ and in $\psi$. Now, since $L_{H}$ extends $L$ and is closed under uniform substitution of formulas for proposition letters, we have:

$$
\begin{gathered}
L_{H} \vdash\left(\bigwedge_{\substack{1 \leq k \leq n \\
\chi \in S u b(\phi)}} \diamond^{+}\left(i_{k} \wedge \chi\right) \rightarrow \square^{+}\left(i_{k} \rightarrow \chi\right)\right) \wedge \phi \rightarrow \theta\left[\overrightarrow{p_{i}} / \vec{i}\right] \\
L_{H} \vdash \theta\left[\overrightarrow{p_{i}} / \vec{i}\right] \rightarrow\left(\bigwedge_{\substack{1 \leq k \leq n \\
\chi \in \operatorname{Sub}(\psi)}} \diamond^{+}\left(i_{k} \wedge \chi\right) \rightarrow \square^{+}\left(i_{k} \rightarrow \chi\right)\right) \rightarrow \psi
\end{gathered}
$$

Since $\diamond^{+}(i \wedge \chi) \rightarrow \square^{+}(i \rightarrow \chi)$ is valid in hybrid logic for any $i$ and $\chi$, it follows that $L_{H} \vdash \phi \rightarrow$ $\theta\left[\overrightarrow{p_{i}} / \vec{i}\right]$ and $L_{H} \vdash \theta\left[\overrightarrow{p_{i}} / \vec{i}\right] \rightarrow \psi$. Finally, as we mentioned above, all proposition letters occurring in $\theta\left[\overrightarrow{p_{i}} / \vec{i}\right]$ occur both in $\phi$ and in $\psi$. We conclude that $\theta\left[\overrightarrow{p_{i}} / \vec{i}\right]$ is an interpolant for $\phi \rightarrow \psi$. 
For any hybrid formula $\phi$, let $\mathbb{P}(\phi)$ and $\mathbb{N}(\phi)$ denote the set of proposition letters respectively nominals occurring in $\phi$.

Definition 4.4 (Uniform interpolation for hybrid logics) A hybrid logic L has uniform interpolation over proposition letters, if for each formula $\phi$ and each finite set of proposition letters $P \subseteq \mathbb{P}(\phi)$, there is a formula $\phi_{P}$ such that

- $\mathbb{P}\left(\phi_{P}\right) \subseteq P$, and

- For all formulas $\psi$, if $\mathbb{P}(\psi) \cap \mathbb{P}(\phi) \subseteq P$ and $\mathbb{N}(\psi) \subseteq \mathbb{N}(\phi)$, then $L \vdash \phi \rightarrow \psi$ iff $L \vdash \phi_{P} \rightarrow \psi$.

In contrast to what one might expect, according to this definition the uniform interpolant $\phi_{P}$ does not apply to formulas $\psi$ that contain nominals not occurring in $\phi$.

Theorem 4.5 Let $L$ be a complete modal logic satisfying one of the following conditions:

(a) L has a master modality and admits simple filtration.

(b) $L$ is axiomatizable using only formulas of modal depth $\leq 1$ and/or closed formulas.

If $L$ has uniform interpolation then $L_{H}$ has uniform interpolation over proposition letters.

Proof: We only prove (a), since (b) is similar.

Let $\phi$ be a hybrid formula, $P \subseteq \mathbb{P}(\phi)$, and let $\mathbb{N}(\phi)=\left\{i_{1}, \ldots, i_{k}\right\}$. Let $P^{\prime}=P \cup\left\{p_{i_{1}}, \ldots, p_{i_{k}}\right\}$. Let $\theta$ be a uniform interpolant in $L$ over $P^{\prime}$ of the formula

$$
\left.\phi^{*}=\phi\left[\vec{i} / \overrightarrow{p_{i}}\right] \wedge \bigwedge_{\substack{1 \leq k \leq n \\ \chi \in S u b\left(\phi\left[\vec{i} / \overrightarrow{p_{i}}\right]\right)}} \diamond^{+}\left(p_{i_{k}} \wedge \chi\right) \rightarrow \square^{+}\left(p_{i_{k}} \rightarrow \chi\right)\right)
$$

We claim that $\theta\left[\overrightarrow{p_{i}} / \vec{i}\right]$ is a uniform interpolant in $L_{H}$ of $\phi$ over $P$. Consider any hybrid formula $\psi$ with $\mathbb{P}(\psi) \cap \mathbb{P}(\phi) \subseteq P$ and $\mathbb{N}(\psi) \subseteq \mathbb{N}(\phi)$. We will show that $L_{H} \vdash \phi \rightarrow \psi$ iff $L_{H} \vdash \theta\left[\overrightarrow{p_{i}} / \vec{i}\right] \rightarrow \psi$.

$[\Rightarrow]$ Suppose $L_{H} \vdash \phi \rightarrow \psi$. Let $\Sigma=\operatorname{Sub}\left(\neg(\phi \rightarrow \psi)\left[\vec{i} / \overrightarrow{p_{i}}\right]\right)$, By Corollary 3.4, we have that the formula

$$
\left(\bigwedge_{\substack{1 \leq k \leq n \\ \chi \in \Sigma}} \diamond^{+}\left(p_{i_{k}} \wedge \chi\right) \rightarrow \square^{+}\left(p_{i_{k}} \rightarrow \chi\right)\right) \rightarrow\left(\phi\left[\vec{i} / \overrightarrow{p_{i}}\right] \rightarrow \psi\left[\vec{i} / \overrightarrow{p_{i}}\right]\right)
$$

is $L$-valid. The same argument as in the proof of Theorem 4.3 shows that

$$
\begin{aligned}
& L \vdash\left(\phi\left[\vec{i} / \overrightarrow{p_{i}}\right] \wedge \bigwedge_{\left.\substack{1 \leq k \leq n \\
\chi \in S u b\left(\phi\left[\overrightarrow{p_{i}} / \vec{i}\right]\right.}\right)} \diamond^{+}\left(p_{i_{k}} \wedge \chi\right) \rightarrow \square^{+}\left(p_{i_{k}} \rightarrow \chi\right)\right) \rightarrow \\
& \left(\bigwedge_{\substack{1 \leq k \leq n \\
\chi \in S u b\left(\psi\left[\overrightarrow{\left.p_{i} / \vec{i}\right]}\right)\right.}} \diamond^{+}\left(p_{i_{k}} \wedge \chi\right) \rightarrow \square^{+}\left(p_{i_{k}} \rightarrow \chi\right)\right) \rightarrow \psi\left[\vec{i} / \overrightarrow{p_{i}}\right] \\
& \chi \in \operatorname{Sub}\left(\psi\left[\overrightarrow{p_{i}} / \vec{i}\right]\right)
\end{aligned}
$$

or, equivalently,

$$
\left.L \vdash \phi^{*} \rightarrow\left(\bigwedge_{\left.\substack{1 \leq k \leq n \\ \chi \in S u b\left(\psi\left[\overrightarrow{p_{i}} / \vec{i}\right]\right.}\right)} \diamond^{+}\left(p_{i_{k}} \wedge \chi\right) \rightarrow \square^{+}\left(p_{i_{k}} \rightarrow \chi\right)\right) \rightarrow \psi\left[\vec{i} / \overrightarrow{p_{i}}\right]\right)
$$

Since $\theta$ is a uniform interpolant for $\phi^{*}$, it follows that

$$
L \vdash \theta \rightarrow\left(\left(\bigwedge_{\substack{1 \leq k \leq n \\ \chi \in S u b\left(\psi \overrightarrow{\left.p_{i} / \vec{i}\right]}\right)}} \diamond^{+}\left(p_{i_{k}} \wedge \chi\right) \rightarrow \square^{+}\left(p_{i_{k}} \rightarrow \chi\right)\right) \rightarrow \psi\left[\vec{i} / \overrightarrow{p_{i}}\right]\right)
$$


Since $L_{H}$ extends $L$ and is closed under uniform substitution of formulas for proposition letters, we have:

$$
L_{H} \vdash \theta\left[\overrightarrow{p_{i}} / \vec{i}\right] \rightarrow\left(\left(\bigwedge_{\substack{1 \leq k \leq n \\ \chi \in S u b(\psi)}} \diamond^{+}\left(i_{k} \wedge \chi\right) \rightarrow \square^{+}\left(i_{k} \rightarrow \chi\right)\right) \rightarrow \psi\right)
$$

Since $\diamond^{+}(i \wedge \chi) \rightarrow \square^{+}(i \rightarrow \chi)$ is valid in hybrid logic for any nominal $i$ and formula $\chi$, it follows that $L_{H} \vdash \theta\left[\overrightarrow{p_{i}} / \vec{i}\right] \rightarrow \psi$.

$[\Leftarrow]$ Suppose $L_{H} \vdash \theta\left[\overrightarrow{p_{i}} / \vec{i}\right] \rightarrow \psi$. Since $\theta$ is a uniform interpolant for $\phi^{*}, L \vdash \phi^{*} \rightarrow \theta$. $L_{H}$ extends $L$ and is closed under uniform substitution, hence

$$
L_{H} \vdash\left(\phi \wedge \bigwedge_{\substack{1 \leq k \leq n \\ \chi \in \operatorname{Sub}(\phi)}} \diamond^{+}\left(i_{k} \wedge \chi\right) \rightarrow \square^{+}\left(i_{k} \rightarrow \chi\right)\right) \rightarrow \theta\left[\overrightarrow{p_{i}} / \vec{i}\right]
$$

Since $L_{H} \vdash \diamond^{+}(i \wedge \chi) \rightarrow \square^{+}(i \rightarrow \chi)$ for any nominal $i$ and formula $\chi$, it follows that $L_{H} \vdash \phi \rightarrow \theta\left[\overrightarrow{p_{i}} / \vec{i}\right]$, and therefore, $L_{H} \vdash \phi \rightarrow \psi$.

It is known that K, GL, S5 and Grz have uniform interpolation (see [16] and [9]). From Theorem 4.5 and the fact that GL and $\mathbf{S 5}$ admit simple filtration, it follows immediately that $\mathbf{K}_{H}, \mathbf{S} \mathbf{5}_{H}$ and $\mathbf{G L}_{H}$ have uniform interpolation over proposition letters. Grz does not admit simple filtration. Nevertheless, we will now show that the construction used in the proof of Theorem 4.5 can be applied to $\mathbf{G r z} \mathbf{z}_{H}$ as well.

Theorem 4.6 $\mathbf{G r z}_{H}$ has uniform interpolation over proposition letters.

Proof: Grz admits filtration in the following manner [3]:

For any formula $\phi$, let $\Sigma_{\phi}=S u b(\{\phi\} \cup\{\diamond(\neg \psi \wedge \diamond \psi): \diamond \psi \in S u b(\phi)\})$. For any model $\mathfrak{M}=(W, R, V)$ based on a Grz-frame $\mathfrak{F}$, let $\mathfrak{M}_{\Sigma_{\phi}}=\left(W / \sim_{\Sigma_{\phi}}, R_{\Sigma_{\phi}}, V_{\Sigma_{\phi}}\right)$, where $[w] R_{\Sigma_{\phi}}[v]$ if $[w]=[v]$ or the following two conditions hold:

1. for every $\diamond \psi \in \Sigma_{\phi}, v \mid=\psi \vee \diamond \psi$ implies $w \models \diamond \psi$, and

2. there exists $\diamond \psi \in \Sigma_{\phi}$ with $w \models \diamond \psi$ and $v \not \models \diamond \psi$.

Then $\mathfrak{M}_{\Sigma_{\phi}}$ is again based on a (finite) Grz-frame, and for all $w \in W$ and $\psi \in \Sigma_{\phi}$, $\mathfrak{M}_{\Sigma_{\phi}},[w] \mid=\psi$ iff $\mathfrak{M}, w \models \psi$.

Now consider again the proof of Theorem 4.5. The crux of the proof lies in the fact that the filtration set $\operatorname{Sub}(\neg(\phi \rightarrow \psi))$ can be split up in two disjoint sets, such that every formula in the first set contains only symbols that occur in $\phi$, and every formula in the second set contains only symbols that occur in $\psi$. As we will now show, the same holds for the filtration set of Grz. To see this, note that

$$
\begin{aligned}
& \Sigma_{\neg(\phi \rightarrow \psi)}=\operatorname{Sub}(\{\neg(\phi \rightarrow \psi)\} \cup\{\diamond(\neg \chi \wedge \diamond \chi) \mid \diamond \chi \in S u b(\neg(\phi \rightarrow \psi))\}) \\
& =\operatorname{Sub}(\{\neg(\phi \rightarrow \psi)\} \cup\{\diamond(\neg \chi \wedge \diamond \chi) \mid \diamond \chi \in S u b(\phi)\} \cup\{\diamond(\neg \chi \wedge \diamond \chi) \mid \diamond \chi \in S u b(\psi)\}) \\
& =\{\neg(\phi \rightarrow \psi), \phi \rightarrow \psi\} \cup S u b(\phi) \cup S u b(\{\diamond(\neg \chi \wedge \diamond \chi) \mid \diamond \chi \in S u b(\phi)\}) \\
& =\{\neg(\phi \rightarrow \psi), \phi \rightarrow \psi\} \cup \Sigma_{\phi} \cup \Sigma_{\psi} \\
& \cup \operatorname{Sub}(\psi) \cup \operatorname{Sub}(\{\diamond(\neg \chi \wedge \diamond \chi) \mid \diamond \chi \in S u b(\psi)\})
\end{aligned}
$$

Hence, every formula in $\Sigma_{\neg(\phi \rightarrow \psi)}$ is a Boolean combination of formulas in $\Sigma_{\phi}$ and $\Sigma_{\psi}$. The same argument as in the proof of Theorem 4.5 shows that $\mathbf{G r z} \mathbf{z}_{H}$ has the uniform interpolation over proposition letters. 
Definition 4.7 For any modal logic $L$, let $L^{H}$ be the closure of

$$
L \cup\left\{\diamond^{n}(i \wedge \phi) \rightarrow \square^{m}(i \rightarrow \phi) \mid i \text { is a nominal, } \phi \text { is a formula and } n, m \in \omega\right\}
$$

under Modus Ponens, Necessitation and Uniform Substitution for proposition letters. ${ }^{3}$

For logics that have a master modality, the set of axioms from Definition 4.7 collapses to the single axiom scheme $\diamond^{+}(i \wedge \phi) \rightarrow \square^{+}(i \rightarrow \phi)$.

Theorem 4.8 Let $L$ be a complete modal logic satisfying one of the following conditions:

(a) L has a master modality and admits filtration.

(b) $L$ is axiomatizable using only formulas of modal depth $\leq 1$ and/or closed formulas.

Then $L_{H}=L^{H}$.

Proof: We only prove (a), since (b) is similar.

That $L^{H} \subseteq L_{H}$ is clear (it suffices to observe that $\diamond^{+}(i \wedge \phi) \rightarrow \square^{+}(i \rightarrow \phi)$ is valid, and that the inference rules preserves validity). In the remainder of the proof, we show that $L_{H} \subseteq L^{H}$.

Suppose $L_{H} \vdash \phi$, for some formula $\phi$ with nominals $i_{1}, \ldots, i_{n}$. Let $\Sigma=\Sigma_{\neg \phi\left[\vec{i} / \overrightarrow{p_{i}}\right]}$. Then by Corollary 3.4, the formula

$$
\left(\bigwedge_{\substack{1 \leq k \leq n \\ \psi \in \Sigma}} \diamond^{+}\left(p_{i_{k}} \wedge \psi\right) \rightarrow \square^{+}\left(p_{i_{k}} \rightarrow \psi\right)\right) \rightarrow \phi\left[\vec{i} / \overrightarrow{p_{i}}\right]
$$

is $L$-valid, and hence

$$
L \vdash\left(\bigwedge_{\substack{1 \leq k \leq n \\ \psi \in \Sigma}} \diamond^{+}\left(p_{i_{k}} \wedge \psi\right) \rightarrow \square^{+}\left(p_{i_{k}} \rightarrow \psi\right)\right) \rightarrow \phi\left[\vec{i} / \overrightarrow{p_{i}}\right]
$$

Since $L \subseteq L^{H}$, we have that

$$
L^{H} \vdash\left(\bigwedge_{\substack{1 \leq k \leq n \\ \psi \in \Sigma}} \diamond^{+}\left(p_{i_{k}} \wedge \psi\right) \rightarrow \square^{+}\left(p_{i_{k}} \rightarrow \psi\right)\right) \rightarrow \phi\left[\vec{i} / \overrightarrow{p_{i}}\right]
$$

$L^{H}$ is closed under uniform substitutions, therefore

$$
L^{H} \vdash\left(\bigwedge_{\substack{1 \leq k \leq n \\ \psi \in \Sigma}} \diamond^{+}\left(i_{k} \wedge \psi\right) \rightarrow \square^{+}\left(i_{k} \rightarrow \psi\right)\right) \rightarrow \phi
$$

By definition, we have that $L^{H} \vdash \diamond^{+}(i \wedge \chi) \rightarrow \square^{+}(i \rightarrow \chi)$ for all $i$ and $\chi$. Since $L^{H}$ is closed under Modus Ponens, we conclude that $L^{H} \vdash \phi$.

Remark 4.9 Several instances of Theorem 4.8 have been proved before. For example, cf. Gargov [8] for $\mathbf{P D L} \mathbf{L}_{H}$ and Gargov and Goranko [7] for $\mathbf{K}_{H}$ with the universal modality.

Remark 4.10 Suppose $L$ is a logic that satisfies our conditions (has a master modality, admits filtrations). Furthermore, suppose $L$ is complete for a frame class $F$. In general we cannot conclude from our results that $L^{H}$ is complete for $F$. All we know is that $L^{H}$ is complete for $\operatorname{Fr}(L)$. $\operatorname{The}$ results of Section 2.3 confirm this. Alternatively, consider the case of GL. As is well known, GL is not only complete for the class of transitive conversely well-founded frames (which it defines), but also for the class of finite transitive irreflexive trees (finite trees for short). By Theorem 4.8our results, we know that $\mathbf{G L}^{H}$ is complete for the class of transitive conversely well-founded frames. As it turns out $\mathbf{G L}^{H}$ is not complete for the class of finite trees: the formula

$$
\diamond(p \wedge \diamond i) \wedge \diamond(q \wedge \diamond i) \rightarrow \diamond(p \wedge \diamond q) \vee \diamond(q \wedge \diamond p) \vee \diamond(p \wedge q)
$$




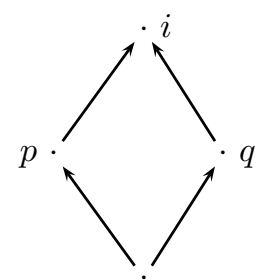

Figure 1: $\mathbf{G L}_{H}$ lacks the tree model property

is valid on finite trees but is not valid on the class of transitive conversely well-founded frames (cf. Figure 1 for a counterexample). Hence, it is not derivable in $\mathbf{G L}^{H}$. We conjecture that if this formula is added as an axiom to $\mathbf{G} \mathbf{L}^{H}$, the resulting logic is complete for finite trees.

\section{Conclusions}

In the paper we provided a translation from hybrid logic to modal logic. We showed that this translation preserves validity for logics with a master modality that admit filtration. Using this translation, we derived transfer results for complexity, interpolation and axiomatic completeness.

As far as we are aware, Corollary 4.1 is the first general complexity result for hybrid logics. Uniform interpolation has also not been studied before in the context of hybrid logic. Finally, while several non-elementary hybrid logics have been investigated before (in particular PDL PL $_{H}$ [13], all general completeness results we are aware of $[7,2,15]$ only apply to elementary logics.

There are still many questions remaining. The study of this topic could be developed in three directions: (1) to find other classes (or extend the class of logics we are working with) for which the translation works. (2) to see which other properties transfer from $L$ to $L_{H}$. Gargov and Goranko [7] and Viana, Marx and ten Cate [15] proved that canonicity transfers. Interesting questions are if the finite model property, decidability, compactness and the Beth property transfer in general (cf. also [7, Problems 4 and 5]). (3) to generalize these results to richer hybrid languages, in particular with satisfaction operators. In fact, some results in this direction will be reported in Part II of this paper.

Finally, note that we could have defined the hybrid companion of a modal logic $L$ to be $L^{H}$ rather than $L_{H}$. In that case, we clearly get axiomatizations for free, and this definition would apply also to incomplete modal logics. On the other hand, transfer of completeness is in this case not straightforward anymore, and indeed an interesting question.

\section{References}

[1] Carlos Areces, Patrick Blackburn, and Maarten Marx. Hybrid logics: Characterization, interpolation and complexity. Journal of Symbolic Logic, 66(3):977-1010, 2001.

[2] Patrick Blackburn, Maarten de Rijke, and Yde Venema. Modal logic. Cambridge University Press, Cambridge, UK, 2001.

[3] George Boolos. The Logic of Provability. Cambridge University Press, 1993.

[4] Balder ten Cate. Interpolation for extended modal languages. Submitted for publication. Available at http://staff.science.uva.nl/ bcate, 2003.

[5] Alexander Chagrov and Michael Zakharyaschev. Modal logic. Oxford University Press, 1997.

\footnotetext{
${ }^{3}$ By Uniform Substitution for proposition letters, we mean that proposition letters (not nominals) can be replaced uniformly by arbitrary formulas [2].
} 
[6] Dov Gabbay, Ágnes Kurucz, Frank Wolter, and Michael Zakharyaschev. Many-Dimensional Modal Logics: Theory and Applications, volume 148 of Studies in Logic. North-Holland, 2003.

[7] George Gargov and Valentin Goranko. Modal logic with names. Journal of Philosophical Logic, 22:607-636, 1993.

[8] Georgi Gargov. The decidability of fundamental combinatory propositional dynamic logic (russian). The mathematical theory of programming (Russian), 170:42-49, 1985. Akad. Nauk SSSR Sibirsk. Otdel., Vychisl. Tsentr, Novosibirsk.

[9] Silvio Ghilardi and Marek Zawadowski. Sheaves, Games and Model Completions. Trends in logic. Kluwer Academic, 2002.

[10] George Goguadze, Carla Piazza, and Yde Venema. Simulating polyadic modal logics by monadic ones. Journal of Symbolic Logic, 68(2):419-462, 2003.

[11] Valentin Goranko and Solomon Passy. Using the universal modality: gains and questions. Journal of Logic and Computation, 2(1):5-30, 1992.

[12] Marcus Kracht and Frank Wolter. Simulation and transfer results in modal logic - a survey. Studia Logica, 59:149-177, 1997.

[13] Solomon Passy and Tinko Tinchev. An essay in combinatory dynamic logic. Information and computation, 93:263-332, 1991.

[14] Schiller Joe Scroggs. Extensions of the Lewis system S5. Journal of Symbolic Logic, 16:111120, 1951.

[15] Petrucio Viana, Maarten Marx, and Balder ten Cate. Canonicity and sahlqvist theory for hybrid logic. Manuscript.

[16] Albert Visser. Uniform interpolation and layered bisimulation. In Gödel 1996, volume 6 of Lecture Notes Logic, pages 139-164, Berlin, 1996. Springer. 